\title{
Toward functional neuronavigation: implementation of functional magnetic resonance imaging data in a surgical guidance system for intraoperative identification of motor and language cortices
}

\author{
Technical note and illustrative case
}

\author{
Geert-Jan Rutten, M.D., Ph.D., Nick Ramsey, Ph.D., Herke-Jan Noordmans, Ph.D., \\ Peter Willems, M.D., Peter van Rijen, M.D., Ph.D., \\ Jan Willem Berkelbach van der Sprenkel, M.D., Ph.D., Max Viergever, Ph.D., \\ and Cees van Veelen, M.D., Ph.D.
}

Departments of Neurosurgery and Psychiatry, and the Image Sciences Institute, University Medical Center Utrecht, The Netherlands

\begin{abstract}
In recent years, surgical navigation systems have become equipped to allow incorporation of data such as functional neuronavigation data. Functional magnetic resonance (fMR) imaging is a noninvasive modality that demonstrates various brain functions. Although still in an experimental stage, fMR imaging is a promising tool for mapping of motor and language functions. One advantage is that it can be implemented in presurgical imaging protocols and is therefore potentially widely available in general neurosurgical practice. In this paper the integration of fMR imaging and surgical navigation is described, and the potential advantages and pitfalls of its application in clinical practice are discussed.
\end{abstract}

KEY WORDS - functional magnetic resonance imaging • electrocortical mapping • image guidance • neuronavigation

In neurosurgery, a number of invasive techniques may be used prior to the resection to delineate brain areas that are indispensable (that is, critical) for normal motor and language function to prevent postoperative functional deficits. These include intraoperative iECS mapping, extraoperative ECS mapping with placement of subdural electrodes, and intraoperative sensory evoked potential monitoring. ${ }^{1,2,6}$ These traditional modalities are limited by several practical and methodological constraints. ${ }^{11}$ One major disadvantage is that the obtained information is not available prior to surgery.

Functional MR imaging is a completely noninvasive method that offers functional information with high spatial (millimeters) and temporal (seconds) resolution. ${ }^{9}$ Other advantages are that fMR imaging is readily implemented on a standard clinical imager and may be included in standard preoperative imaging protocols. It can be performed to aid in presurgical planning, eliminate the added time and inconvenience of current intraoperative mapping

Abbreviations used in this paper: $\mathrm{ECS}=$ electrocortical stimulation; $\mathrm{fMR}$ = functional magnetic resonance; FOV = field of view; iECS $=$ intraoperative ECS; 3D = three-dimensional. techniques, or avoid the additional operation that is required in cases requiring stimulation via subdural electrodes.

In recent years, image-guided systems have been used for intraoperative navigation based on preoperatively acquired CT-scanning-and MR imaging-documented structural information. ${ }^{17}$ This offers the possibility of adding functional information to these systems, allowing for "functional neuronavigation;" thus, the surgeon can rely on intraoperative structural data (location of the lesion) and functional data (location of indispensable functional areas). ${ }^{3,4,8,10,18}$ During the past 3 years, we have incorporated information from fMR imaging into a surgical guidance system for intraoperative localization of motor areas in 15 patients and language areas in 18 patients. In this paper we describe our method and illustrate its potential advantages in surgical practice. Functional MR imaging information was validated using iECS mapping in these patients as part of experimental studies; results of these findings are reported elsewhere and are beyond the scope of this paper. ${ }^{15,16}$ To illustrate the procedures we present a case study. A brief discussion on the indications and current pitfalls of functional neuronavigation for critical motor areas is given. 


\section{ILLUSTRATIVE CASE}

Examination. This 32-year-old, right-handed woman presented with generalized seizures. Results of neurological examination were normal; her medical history was significant for migraine. Cranial MR imaging revealed a large hypodense, nonenhancing space-occupying mass in the right frontal lobe, which was suspected to be a lowgrade glioma (Fig. 1). She was started on a course of valproic acid to control the symptomatic epilepsy. Prior to surgery, she underwent a battery of fMR imaging-based sensorimotor tasks to identify the primary motor cortex and to assess whether complete tumor resection was feasible without a significant risk of postoperative motor deficit.

\section{MATERIALS AND METHODS}

\section{Functional MR Imaging}

One month before surgery, fMR imaging is performed using a 3D blood oxygen level-dependent technique (navigated PRESTO sequence, TE $36 \mathrm{msec}$, TR $24.5 \mathrm{msec}$, flip angle $10^{\circ}$, matrix $52 \times 64 \times 26$, FOV $183 \times 225 \times$ 91 , voxel size $3.51 \mathrm{~mm}$ isotropic, scan time 2.4 seconds), implemented on a Philips ACS-NT 1.5 T imager with PT6000 gradients (Philips Medical Systems, Best, The Netherlands). Functional images are aligned perpendicularly to the course of the central sulcus and included the frontal and parietal lobe.

To minimize head motion, the patient's head is restrained using a strap and foam pads. The anatomical image covers the whole head, so that we can subsequently use a set of the following eight anatomical reference points for registration during surgery: left and right ear, inner and outer canthus of both eyes, nasion, and tip of the nose. We use anatomical reference points because fMR imaging is usually performed several weeks prior to surgery. For practical reasons, adhesive skin markers are used only when fMR imaging is performed 1 or 2 days before surgery. The method involving anatomical markers is only slightly more inaccurate than that requiring adhesive markers (on average, a few millimeters). ${ }^{21}$ Care is taken that this set of anatomical reference points is not displaced or distorted by the strap or foam pads.

The following four tasks are used in the imaging system (according to a standardized experimental protocol): movement of the left and right hand, tactile stimulation of the left hand, and movement of the left foot. These tasks are practiced prior to the actual imaging session. Per task, 252 fMR imaging volumes are acquired such that periods of activity (for example, movements or tactile stimulation) are alternated with rest periods ( 22 seconds' duration each). In the imaging room, one of the investigators instructs the patient before each block and then monitors the patient's performance. During the motor task, a green or red dot is projected on a screen that the patient can see on a headcoil-mounted mirror. The active condition for motor hand movements consists of pushing the button of a box with the thumb, in pace with the flashing green dot at 2 Hz. The red nonflashing dot indicates the rest condition. For movement of the foot, the frequency of the flashing dot is set at $1 \mathrm{~Hz}$. Here, the patient makes alternating flexion and extension movements with toes and ankle during

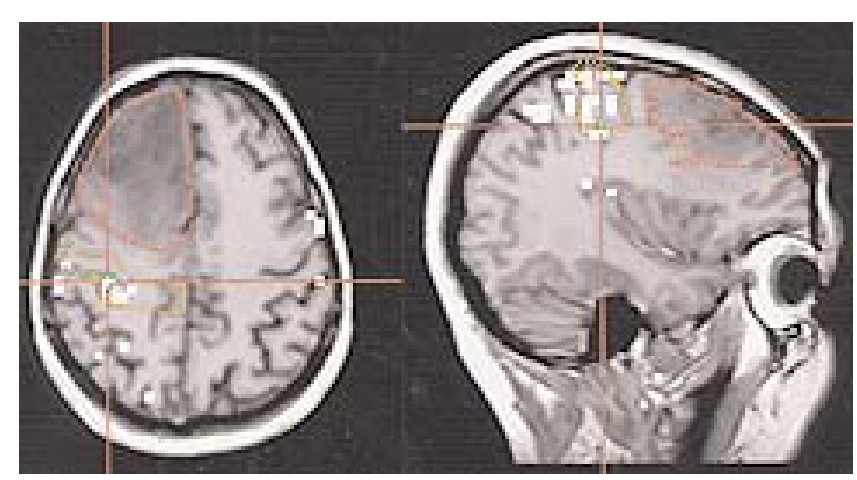

Fig. 1. Axial (left) and sagittal (right) images of the 3D image data set used for neuronavigation in the illustrative case, a 32-yearold woman with a right frontal glioma (images obtained using the surgical MKM workstation). Information from fMR imaging (reflecting left-hand movement) is shown in white voxels. Using software on the surgical planning station, contours were drawn around the tumor (red), primary motor cortex (orange), and fMR imaging areas (green). As judged from fMR imaging data, there is a margin of approximately $1 \mathrm{~cm}$ between the primary motor cortex and the posterior border of the tumor.

the active condition. The leg is supported by a pillow to avoid motion during this task. For tactile stimulation, the investigator lightly brushes the palm of the left hand (median nerve territory, paced at $1 \mathrm{~Hz}$ ) while the patient has his/her eyes closed.

\section{Structural MR Imaging}

At the end of the fMR imaging session, an anatomical volume is obtained for $3 \mathrm{D}$ visualization of fMR imaging results, as well as for intraoperative navigation involving the surgical guidance system (3D-fast field echo, TE 4.6 msec, TR $30 \mathrm{msec}$, flip angle $30^{\circ}$, FOV $256 \times 256 \times 150$, voxel size $1 \times 1 \times 1.2 \mathrm{~mm}$ ).

\section{Registration of Functional and Anatomical Data}

Data are transferred from the scanner to a HewlettPackard Workstation for further processing by using custom-made programs in PV-WAVE-based software. All functional volume images (total 1008) are motion corrected and registered to the anatomical image to determine location of activity; see also the study by Ramsey and colleagues. ${ }^{14}$ For the registration procedure, it is essential that at least one functional volume image is acquired using a different flip angle $\left(30^{\circ}\right)$ than the normal functional volume images. This alternate image has characteristics of both functional and anatomical images and can therefore serve as an intermediate to which both the later images are registered. Registration consists of three steps. 1) Registration of anatomical and $30^{\circ}$ functional images is performed. Because the internal contrast of the $30^{\circ}$ image resembles that of an anatomical image, this match is usually excellent. The accuracy of registration results is verified by visual inspection of the outline of the $30^{\circ}$ functional image superimposed onto the registered anatomical image. 2) The last functional volume image is registered to the $30^{\circ}$ functional image. Because functional volume images have virtually no internal contrast (that is, no gray/ white matter differentiation), automated registration is 
done by the matching of brain tissue contours. Verification of the registration procedure is conducted by visual inspection of the outline of the registered last functional image superimposed onto the $30^{\circ}$ functional image. 3) All other functional images are registered to the last functional volume image that is registered to $30^{\circ}$ functional im-age. As in Step 2, automated registration is performed by matching of brain tissue contours. Verification of the registration procedure is conducted by inspection of all registered functional images in a cine-loop, which allows for identification of possible mismatches of individual images, as well as for fMR image quality (for example, motion artifacts). This procedure is widely used in our fMR imaging laboratory, and yields excellent results in almost all experiments. In the rare cases in which matching fails, a manual preregistration match solves the problem.

\section{Statistical Procedures}

After coregistration of functional and anatomical images, statistical maps for each task are obtained using a multiple regression algorithm that includes an activity factor (on-off function) and detrending factors. ${ }^{22}$ This analysis results in a statistical $t$ value per voxel for each task (Fig. 2 upper). Next, statistical maps are thresholded; voxels are considered "active" if the t value exceeds a critical threshold. For each task, brain activity maps are created at two different thresholds; 3.5 and 4.5. The latter value corresponds to a probability value less than 0.05 after Bonferroni correction for the number of comparisons (that is, the number of voxels in the imaged volume) and is therefore the statistically correct value. ${ }^{20}$ During surgery we additionally localize fMR imaging areas that are active at the lower (3.5) threshold to increase sensitivity for functional areas. No "smoothing" of functional images is applied-as in our experience, critical structures for motor or language function tend to be small.

Although, obviously, there is no method to assess whether a voxel is truly "activated," it is important to check that lack of activation is not due to artifacts that occur during imaging or postprocessing of data. Because there are no undisputed quantitative measures that reflect the quality of fMR imaging results, we rely on visual inspection of statistical maps ( $t$ value/voxel) and maps that show the standard deviation and the residual statistical error per voxel (Fig. 2). In addition, some quantitative measures regarding image stability are calculated; the image technique is very effective in removing image distorting effects of motion during scans. ${ }^{14}$

\section{Implementation of Functional Data into the Guidance System}

To incorporate fMR imaging data into the surgical guidance system (MKM, Carl Zeiss, Oberkochen, Germany), a data volume is created that consists of MR imaging information that is superimposed on the anatomical image. Activated fMR imaging voxels are given a value that is 1.1-fold higher than the voxel with the highest value in the anatomical image. In this way, the image retains good contrast while the voxels are clearly displayed (Fig. 1). The combined functional-anatomical data set is transferred via a network to the Zeiss surgical planning work-

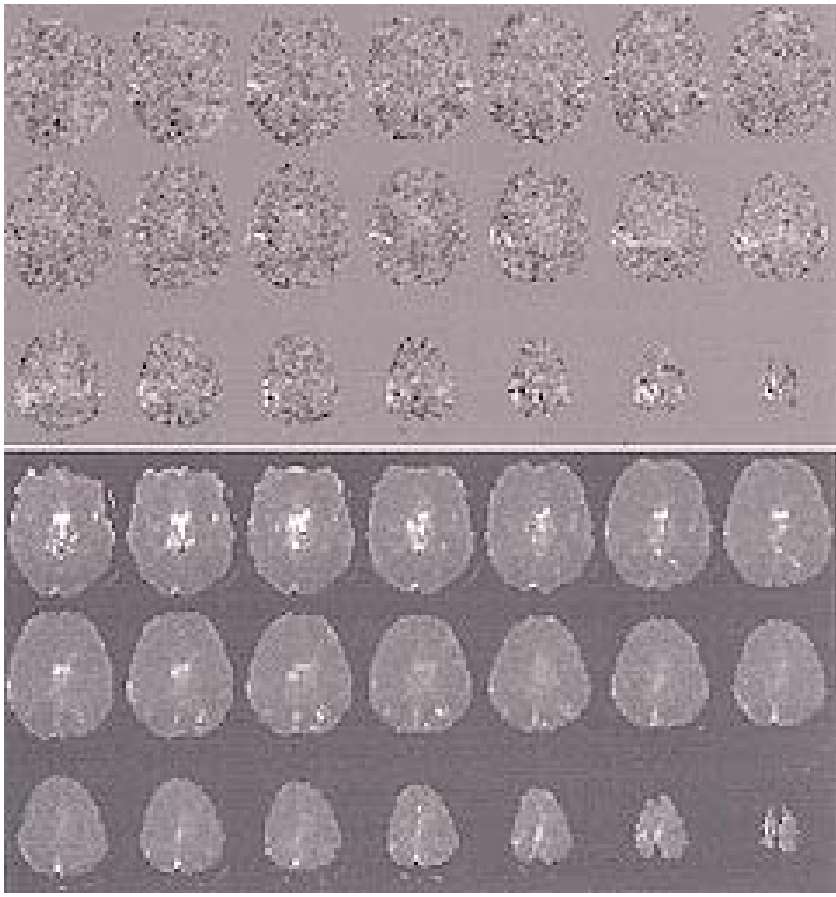

Fig. 2. Axial images demonstrating some of the statistical results corresponding to the task requiring the patient to make repetitive movements of her left hand. Upper: Map showing the statistical $t$ value per voxel. The $t$ values are converted to a gray scale, ranging from black (negative values) to white (positive values). Lower: Map showing the residual error per voxel — that is, the percentage of the signal that can not be explained by the terms in the regression analysis. The whiter voxels show the areas of relatively high image instability.

station for further processing. A custom-made software program replaces the data of an existing image file with the functional-anatomical data set acquired in the patient.

\section{Presurgical Planning}

Prior to the operation, fMR imaging voxels in the vicinity of the tumor, as well as the tumor itself, are indicated by colored contours (Fig. 1). Functional MR imaging voxels are contoured for verification with ECS map. In addition, the primary motor cortex is contoured on the basis of functional (fMR imaging information) and anatomical characteristics (identification of the precentral gyrus [see Yousry and colleagues ${ }^{23}$ and Berger and colleagues ${ }^{1}$ for details]). With the Zeiss MKM surgical guidance system, the contours preplanned on the surgical workstation can be visualized within the surgical field of the operation microscope (Figs. 3 and 4B). Also, the navigated robotic arm of the MKM can automatically guide the focus of the microscope to preplanned targets or contours (for example, the primary motor cortex [Fig. 4B]).

\section{Surgical Procedures}

After induction of general anesthesia, the patient is positioned appropriately for the planned procedure. No long-term muscle relaxants are used in view of motor cortex mapping. Special attention is given to the fact that the left arm, hand, and foot can be observed for movements 


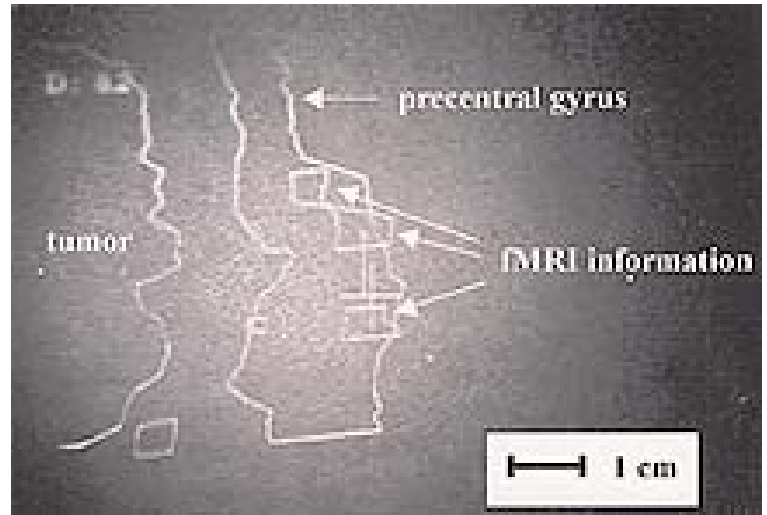

Fig. 3. Preoperative view through the operation microscope (actual view in color). Anatomical and functional information that was previously indicated on the surgical planning station is now projected on the patient's skin for planning of the area of trephination. Orientation and FOV are comparable to the area displayed within the white box in Fig. 4A (that is, part of the right frontoparietal area). The visible contours represent the posterior part of the tumor, the precentral gyrus, and functional MR imaging data (left motor hand function). Note the margin of approximately $1 \mathrm{~cm}$ between the tumor and the primary motor cortex.

during iECS mapping. The patient's head is immobilized in a Mayfield headrest system (OMI, Inc., Cincinnati, $\mathrm{OH})$, and patient-to-image registration was established using a rigid body transformation based on the previously described anatomical landmarks. ${ }^{5}$ Once the accuracy of the patient-to-image registration had been confirmed by multiple landmark tests, the preplanned contours are displayed in the surgeon's viewing field to plan the area that had to be exposed during surgery (Fig. 3). Next, a right frontoparietal craniotomy is made and the cortical surface is exposed.

Prior to iECS, the surgical guidance system is used to mark the cortical localization of the labeled fMR imaging areas (or projection to the cortical surface in case in which these areas are located beneath the surface or within a sulcus) with sterile numbered tags directly after cortical exposure (Fig. 4B). As a result of craniotomy and dural opening alone, errors up to $10 \mathrm{~mm}$ have been reported for image-guided neuronavigational systems because of peroperative shifting of brain structures. ${ }^{7}$ The position of the sterile tags is therefore checked using a volume rendering of the cortical surface that contained the fMR image data. Because the cortical topography is relatively unaffected by brain shift, the rendering is considered the gold standard for localization of the selected fMR imaging areas. Figure 4B shows that the preplanned contour of the motor cortex (precentral gyrus) corresponds to the outline of a gyrus found intraoperatively and to a gyrus visible on the cortex rendering (Fig. 4C). The estimated spatial difference between the virtual and real outline of this gyrus is 4 $\mathrm{mm}$ (that is, the inaccuracy of the surgical guidance system). Using this information, the positions of the fMR imaging targets, as indicated by the navigator, can be slightly corrected (a few millimeters, on average).

\section{Intraoperative ECS Mapping}

The iECS mapping procedure is performed after induc-

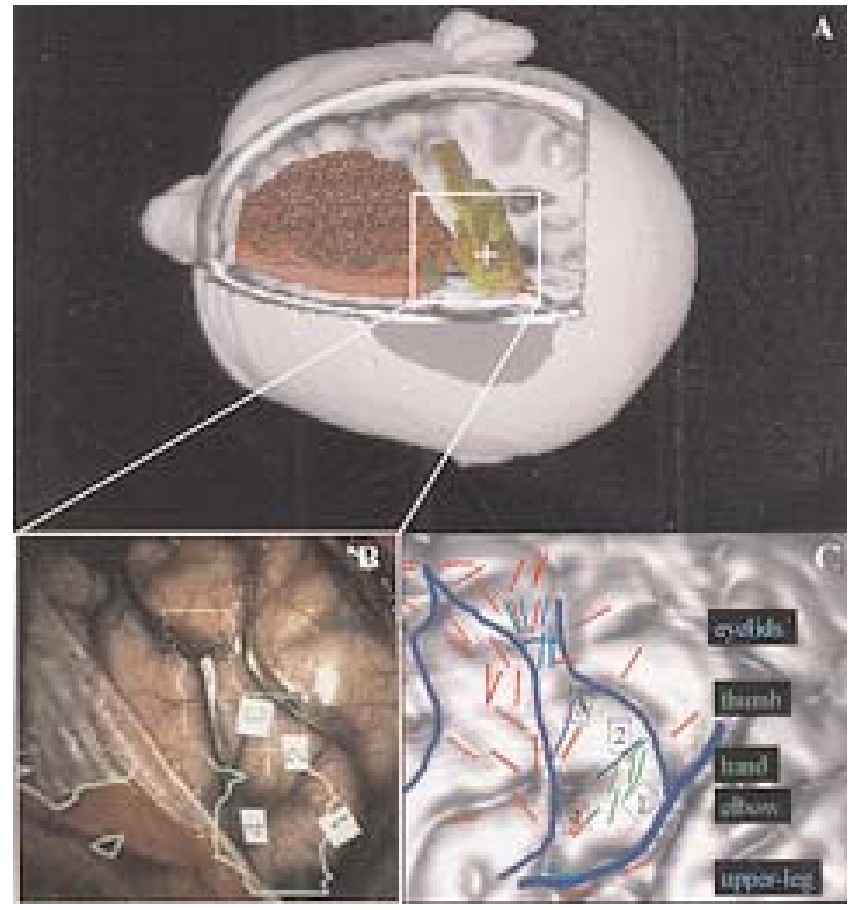

Fig. 4. A: Top view of the patient's head in a volume rendering of MR images. In the right hemisphere, contours of the frontal tumor (red), the precentral gyrus (orange), and functional areas are shown. B: Intraoperative view (corresponding to the area within the white box in A) seen through the operating microscope, demonstrating the exposed cortical surface. The cross represents the focal point of the microscope and corresponds with that in A. The contour represents the primary motor cortex as planned prior to the operation; four numbered tickets have been placed over the motor gyrus, based on fMR imaging information, by using the surgical guidance system. C: Volume rendering of the cortical surface of the area shown in B, demonstrating that accurate MR imagingbased identification of cortical topography is feasible. Large intrasulcal veins are indicated in blue. Electrocortical stimulation mapping confirmed the location of the primary motor cortex; the location and results of ECS are indicated with the colored bars: green bars, motor movements of left arm/hand; light blue bars, eyelid movement; dark blue bar, upper-leg movement; red bars, no motor movements. A macroscopic resection of the tumor was performed and no postoperative neurological deficits developed.

tion of general anesthesia; details have been described elsewhere. ${ }^{15}$ Electrocortical stimulation covered the contoured gyrus (precentral gyrus), as well as the adjacent gyri located anteriorly and posteriorly. During stimulation electrocorticography was performed to check for possible afterdischarges or epileptic seizures, which could result in biased stimulation responses. The entire stimulation procedure was recorded by a video camera that was mounted on the operation lamp, to allow for detailed comparison of fMR imaging and iECS mapping results afterward (data not reported in this paper). After iECS mapping, standard resection was conducted.

\section{RESULTS IN AN ILLUSTRATIVE CASE}

Operation. None of the functional volumes was excluded from analysis. All fMR imaging-based tasks yielded 
activation patterns at the highest threshold $(\mathrm{t}>4.5)$. Based on fMR imaging results, we could unequivocally determine the gyrus that represented the "primary motor cortex," and this was confirmed ECS mapping (Fig. 4). A margin of one gyrus $(\sim 1 \mathrm{~cm})$ was identified between the primary motor cortex and the posterior border of the tumor, suggesting that a radical resection of the tumor was possible without major risk of a postoperative hemiparesis.

Postoperative Course. A complete resection of the tumor was performed, and there were no postoperative neurological deficits. Histological diagnosis was an anaplastic astrocytoma (Grade III), and the patient underwent radiation therapy (60 Gy). Follow-up MR imaging over a 2 -year period has revealed no residual or recurrent of tumor.

\section{DISCUSSION}

The authors of previous studies have shown that the primary motor cortex can be reliably located with anatomical landmarks in patients in whom no space-occupying abnormalities exist in the central region. ${ }^{23}$ Functional mapping becomes necessary when the normal topography is distorted or when there is suspicion that reorganization has shifted functions to another location. It must be noted that in patients with developmental disorders or a brain lesion acquired in early life, "normal" cortical anatomy of the pre- and postcentral gyri on MR images or visual inspection is not a guarantee that these structures remain involved in critical sensorimotor function. In these cases, anatomical landmarks cannot be fully trusted as indicators of the location of the primary sensorimotor cortex. Another indication for functional motor mapping is to identify the spatial relationship between areas that control the arm and hand, as well as areas that represent face and tongue musculature. Resection of these latter areas can be performed with little or no morbidity (presumably due to a bilateral cortical representation ${ }^{19}$ ), whereas damage to areas that control the extremities consistently leads to a persistent and grave hemiparesis. ${ }^{6,12,24}$ There are currently no studies that have validated fMR imaging for its ability to separate motor areas of different functions along the primary motor cortex.

The accepted standard for functional surgery near the motor cortex involves intraoperative cortical mapping, as already described by Penfield and Boldrey in 1937. ${ }^{13}$ With the advent of surgical guidance systems that allow incorporation of functional data, however, the possibility of a noninvasive alternative arose. The merits of this new technology are obvious; the greatest advantage would be that functional information is more readily available and can be used in general neurosurgical practice, where it may be used to avoid unnecessary intrusions into the eloquent cortex and avoid undesirable limited resections of tumors or arteriovenous malformations.

We found that implementation of fMR imaging information in a surgical guidance system is relatively straightforward. In its simplest form (as presented here), functional neuronavigation only requires that the data volume normally read into the surgical guidance system contains
fMR imaging information. Because we acquire anatomical and functional information in one session, no additional registration of fMR imaging data to MR imaging data for neuronavigation is necessary, and this prevents an additional registration error.

If functional neuronavigation is to be used as a clinical tool for localization of indispensable areas for motor (or other cognitive) functions, two main problems remain to be solved. First, fMR imaging methodology has to be standardized, and the resulting brain activity maps that are thought to reflect a particular function need to be validated in detail against accepted clinical modalities for this purpose, as well as patient outcome. Second, because of brain shift during surgery (for example, after opening of the skull and dura mater, or during tumor resection), an error can occur in localization of the navigated fMR imaging information (which is linked to the anatomical image that is obtained prior to surgical procedures). To compensate for possible errors of this type, we currently use a rendering of the patient's cortical surface (that includes fMR imaging information) that we compare with the actual morphological characteristics of the patient's cortex. With the combination of the surgical guidance system and the cortical rendering, we are able quickly to localize a set of preplanned (functional) targets and avoid large errors due to brain shift.

The acquisition of fMR imaging activation maps is by far the most time-consuming factor in the process of functional neuronavigation. Once these imaging maps have been obtained, their data can be implemented relatively easily in a surgical guidance system and can assist the surgeon in maximizing the benefits of the resection while minimizing the risk of surgery. Because there are to date no standardized fMR imaging protocols that can selectively identify areas critical for a particular sensorimotor or cognitive function, however, proper interpretation of the resulting brain maps still requires the experience of the investigators.

\section{References}

1. Berger MS, Cohen WA, Ojemann GA: Correlation of motor cortex brain mapping data with magnetic resonance imaging. J Neurosurg 72:383-387, 1990

2. Berger MS, Kincaid J, Ojemann GA, et al: Brain mapping techniques to maximize resection, safety, and seizure control in children with brain tumors. Neurosurgery 25:786-792, 1989

3. Cosgrove GR, Buchbinder BR, Jiang H: Functional magnetic resonance imaging for intracranial navigation. Neurosurg Clin N Am 7:313-322, 1996

4. Gallen CC, Sobel DF, Waltz T, et al: Noninvasive presurgical neuromagnetic mapping of somatosensory cortex. Neurosurgery 33:260-268, 1993

5. Germano IM, Villalobos H, Silvers A, et al: Clinical use of the optical digitizer for intracranial neuronavigation. Neurosurgery 45:261-270, 1999

6. Gregorie EM, Goldring S: Localization of function in the excision of lesions from the sensorimotor region. J Neurosurg 61: 1047-1054, 1984

7. Hill DL, Maurer CR Jr, Maciunas RJ, et al: Measurement of intraoperative brain surface deformation under a craniotomy. Neurosurgery 43:514-528, 1998

8. Jannin P, Morandi X, Fleig OJ, et al: Integration of sulcal and 
functional information for multimodal neuronavigation. J Neurosurg 96:713-723, 2002

9. Moonen CT, Bandettini PA: Functional MRI. Berlin Heidelberg: Springer-Verlag, 2000

10. Nimsky C, Ganslandt $\mathrm{O}$, Kober $\mathrm{H}$, et al: Integration of functional magnetic resonance imaging supported by magnetoencephalography in functional neuronavigation. Neurosurgery 44: 1249-1256, 1999

11. Ojemann GA: Brain organization for language from the perspective of electrical stimulation mapping. Behav Brain Sci 6: 189-230, 1983

12. Olivier A: Surgical strategies for patients with supplementary sensorimotor area epilepsy. The Montreal experience Adv Neurol 70:429-443, 1996

13. Penfield W, Boldrey E: Somatic motor and sensory representation in the cerebral cortex of man as studied by electrical stimulation. Brain 60:389-443, 1937

14. Ramsey NF, van den Brink JS, van Muiswinkel AM, et al: Phase navigator correction in 3D fMRI improves detection of brain activation: quantitative assessment with a graded motor activation procedure. Neuroimage 8:240-248, 1998

15. Rutten GJ, Ramsey NF, van Rijen PC, et al: Development of a functional magnetic resonance imaging protocol for intraoperative localization of critical temporoparietal language areas. Ann Neurol 51:350-360, 2002

16. Rutten GJ, van Rijen PC, van Veelen CW, et al: Language area localization with three-dimensional functional magnetic resonance imaging matches intrasulcal electrostimulation in Broca's area. Ann Neurol 46:405-408, 1999

17. Schneider JP, Schulz T, Schmidt F, et al: Gross-total surgery of supratentorial low-grade gliomas under intraoperative MR guidance. AJNR 22:89-98, 2001

18. Schulder M, Maldjian JA, Liu WC, et al: Functional imageguided surgery of intracranial tumors located in or near the sensorimotor cortex. J Neurosurg 89:412-418, 1998

19. Taylor L, Jones L: Effects of lesions invading the postcentral gyrus on somatosenory thresholds on the face. Neuropsychologia 35:953-961, 1997

20. van Gelderen P, Ramsey NF, Liu G, et al: Three-dimensional functional magnetic resonance imaging of human brain on a clinical 1.5-t scanner. Proc Natl Acad Sci USA 92:6906-6910, 1995

21. Willems PWA, Berkelbach van der Sprenkel JW, Tulleken CAF: Comparison of adhesive markers, anatomical landmarks and surface matching in patient-to-image registration for frameless stereotaxy. Proc SPIE 4158:156-163, 2001

22. Worsley KJ, Friston KJ: Analysis of fMRI time-series revisited-again. Neuroimage 2:173-181, 1995

23. Yousry TA, Schmid UD, Alkadhi H, et al: Localization of the motor hand area to a knob on the precentral gyrus. A new landmark. Brain 120:141-157, 1997

24. Zentner J, Hufnagel A, Pechstein U, et al: Functional results after resective procedures involving the supplementary motor area. J Neurosurg 85:542-549, 1996

Manuscript received May 14, 2003.

Accepted in final form June 13, 2003.

Address reprint requests to: Geert-Jan M. Rutten, M.D., Ph.D., Department of Neurosurgery, University Medical Center Utrecht, 